\title{
Study of stellar population in the new extended star formation region associated with five IRAS sources
}

\author{
N.M.Azatyan* and E.H. Nikoghosyan \\ NAS RA V. Ambartsumian Byurakan Astrophysical Observatory (BAO), Armenia \\ *E-mail: nayazatyan@bao.sci.am
}

\begin{abstract}
We present the investigation results of structure and stellar content of a molecular cloud surrounding the five IRAS sources: $05168+3634$, $05184+3635,05177+3636,05162+3639$, and IRAS $05156+3643$. Using multi-color criteria, we identified a rich population of embedded YSO candidates with infrared excess (Class $0 / \mathrm{I}$ and Class II) and their characteristics in a quite large molecular cloud which is located in the region of 24 arcmin radius. The molecular cloud includes 240 candidates of YSOs within the radii of sub-regions around 5 IRAS sources. The color-magnitude diagrams of the sub-regions suggest a very young stellar population. We construct the K luminosity function (KLF) of the sub-regions and according to the values of the slopes of KLFs, the age of the sub-regions can be estimated between 0.1-3 Myr. The SEDs are constructed for 45 Class I and 75 Class II evolutionary stages YSOs and the received parameters of those YSOs are well correlated with the results obtained by other methods.
\end{abstract}

Keywords: stars: pre-main sequence - stars: luminosity function - infrared: stars - radiative transfer - individual objects: IRAS 05168+3634, IRAS 05184+3635, IRAS 05177+3636, IRAS 05162+3639, IRAS 05156+3643

\section{Introduction}

In this paper, we present a detailed study of a extended star formation region surrounding IRAS $05168+3634$ source. IRAS $05168+3634$ is also known as Mol 9 from the catalogue of Molinari et al. (1996). Within 2 arcmin radius from IRAS $05168+3634$ source, it has been detected three objects with magnitudes of MSX survey (Egan et al. 2003), one of which is associated with IRAS $05168+3634$ source.

IRAS $05168+3634$ is a luminous YSO with estimated $\mathrm{L}=24 \times 10^{3} \mathrm{~L}_{\odot}$ (Varricatt et al. 2010) and it is located in a high-mass star-forming region 
in the pre-UC HII phase (Wang et al. 2009). This star-forming region associated with $\mathrm{H}_{2} \mathrm{O}, \mathrm{NH}_{3}, 44 \mathrm{GHz} \mathrm{CH}_{3} \mathrm{OH}$, as well as $1665 \mathrm{MHz}$ and $1667 \mathrm{MHz}$ $\mathrm{OH}$ maser emission (Zhang et al. 2005, Molinari et al. 1996, Fontani et al. 2010, Ruiz-Velasco et al. 2016), the $\mathrm{SiO}(\mathrm{J}=2-1)$ line (Harju et al. 1998), and four ${ }^{13} \mathrm{CO}$ cores (Guan et al. 2008b). Molinari et al. (1998) have detected 6-cm radio emission 102 arcsec away from the IRAS $05168+3634$ source.

The embedded stellar cluster in this region was detected in the NIR and MIR by various authors (Kumar et al. 2006; Faustini et al. 2009; Azatyan et al. 2016). In (Azatyan et al. 2016) it was showed that this is a bimodal cluster with 1.5 arcmin radius from geometric center of the cluster which does not coincide with the IRAS $05168+3634$ source. One of the subgroups is concentrated around the IRAS $05168+3634$ source and one must notice, it does not contain a rich population compared to other concentration.

The distance estimations of this region are different. A kinematic distance was estimated $6.08 \mathrm{kpc}$ (Molinari et al. 1996) based on systemic LSR velocity $\mathrm{V}_{L S R}=-15.5 \pm 1.9 \mathrm{~km} / \mathrm{s}$. The trigonometric parallax of IRAS $05168+3634$ with VERA is $0.532 \pm 0.053$ mas, which is corresponding to a distance of $1.88_{-0.17}^{+0.21} \mathrm{kpc}$ placing the region in the Perseus arm, rather than the Outer arm (Sakai et al. 2012). This large difference of estimated distances causes some significant differences of physical parameters for individual members.

The observational data used to make the subsequent analysis are discussed in Section 2. The results and discussion are given in Section 3 and Section 4 concludes the results of this work.

\section{Archival data}

For our study, we have used the data covering a wide infrared range from NIR to FIR wavelength. At first, it is the NIR photometric data and images of UKIRT Infrared Deep Sky Survey (UKIDSS) Galactic Plane Survey DR6 (GPS, Lucas et al. 2008) in J, H, and K bands.

MIR observations of this region have obtained using the Spitzer Space Telescope under the Galactic Legacy Infrared Midplane Survey Extraordinaire 360 (GLIMPSE 360) program (Churchwell et al. 2009) in the 3.6, $4.5 \mu \mathrm{m}$ bands with the resolution of $0.6^{\prime \prime} / \mathrm{px}$. We have also used the data of Wide-field Infrared Survey Explorer (WISE, Wright et al. 2010) $(3.4 \mu \mathrm{m}$, $4.6 \mu \mathrm{m}, 12 \mu \mathrm{m}$ and $22 \mu \mathrm{m})$ and the MSX survey $(8.28 \mu \mathrm{m}, 12.13 \mu \mathrm{m}, 14.65 \mu \mathrm{m}$ and $21.3 \mu \mathrm{m}$ ).

We obtained FIR data using the instruments Photodetector Array Camera and Spectrometer (PACS, Poglitsch et al. 2010) and Spectral and Photometric Imaging Receiver (SPIRE, Griffin et al. 2010) on the $3.5 \mathrm{~m}$ Herschel Space Observatory (Pilbratt et al. 2010). For our analyses, we used the 
PACS $70,160 \mu \mathrm{m}$ and the SPIRE 250,350 and $50 \mu \mathrm{m}$ photometric data and images with resolutions varying $\backsim 5.5^{\prime \prime}-36^{\prime \prime}$. We also used photometric data of IRAS mission (12 and $25 \mu \mathrm{m})$.

\section{Results and discussion}

\subsection{Structure of the molecular cloud}

Comparison of images taken in different spectral ranges showed that IRAS 05168+3634 star-forming region has more complicated structure in the FIR wavelengths than in the NIR and MIR. Figure 1 shows the region in different wavelengths. Moving toward longer wavelengths, the cloud filaments surrounding IRAS $05168+3634$ source become more expressive and it is obvious that the IRAS $05168+3634$ star-forming region is not limited in 1.5 arcmin radius from geometric center (Azatyan et al. 2016) but is more extended and is located within a 24 arcmin radius molecular cloud where the center of molecular cloud is conditionally selected IRAS $05168+3634$ source. Studying common star-forming region in the molecular cloud, it turns out that apart from IRAS $05168+3634$, there are four IRAS sources: IRAS 05184+3635, IRAS 05177+3636, IRAS 05162+3639, and IRAS 05156+ +3643 , which are embedded in the same molecular cloud. There is very few information about these IRAS sources. IRAS $05184+3635$ and IRAS $05177+$ +3636 are associated with dark clouds DOBASHI 4334 and 4326, respectively (Dobashi 2011). In Casoli et al. (1986), the distances of IRAS 05184+ +3635 and IRAS $05177+3636$ were assessed based on the ${ }^{13} \mathrm{CO}$ velocities: $17 \mathrm{~km} / \mathrm{s}$ and $-15 \mathrm{~km} / \mathrm{s}$ respectively, as a result of which both IRAS $05184+3635$ and IRAS $05177+3636$ were evaluated at the same $1.4 \mathrm{kpc}$ distance. The latter value coincides with the distance of IRAS $05168+3634$ based on trigonometric parallax. This fact also indicates that these IRAS sources are most likely to be found in the same molecular cloud. There are 2 objects near to IRAS $05177+3636$ source detected in submillimeter wavelength (Di Francesco et al. 2008). IRAS $05162+3639$ associated with $\mathrm{H}_{2} \mathrm{O}$ maser (Sunada et al. 2007). It has been detected a high proper-motion star in LSPM-NORTH catalog by 0.35 arcmin far away from the IRAS $05156+3643$ (Lépine \& Shara 2005), which is probably compatible with IRAS $05156+3643$ source within error bar.

We have constructed map of the distribution of stellar surface density within a $48^{\prime} \times 48^{\prime}$ region to investigate the structure of each concentration in the molecular cloud, using photometric data of Herschel PACS Point Source Catalog: $160 \mu \mathrm{m}$ and Extended Source List (red band). The density was determined simply by dividing the number of stellar objects in $200^{\prime \prime} \times 200^{\prime \prime}$ area with step size $100^{\prime \prime}$. Figure 2 shows the map of the distribution of stellar surface density based on Herschel PACS $160 \mu \mathrm{m}$ photometry.

It is known that in the case of homogeneous distribution of stars in the 

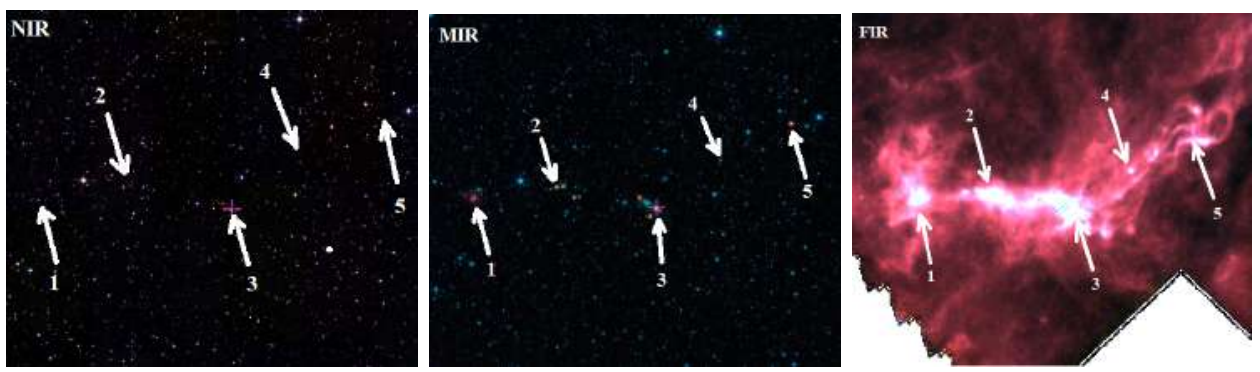

Figure 1: Investigated region in different wavelengths from NIR to FIR. The positions of 5 IRAS sources are marked by arrows and the IRASs are numbered as follows 1-IRAS 05184+3635, 2-IRAS 05177+3636, 3-IRAS 05168+3634, 4IRAS $05162+3639$ and 5-IRAS 05156+3643.

field, the number of stars is comparable to the surface area occupied by those stars. Let us figure out how much the distribution of stars in this field differs from the homogeneous distribution. We can clearly distinguish five subgroups, the distribution of which can not be accidental. From this, one can conclude that all 5 IRAS star-forming regions with high probability belong to the same molecular cloud and, therefore, are at the same distance. As the distances for whole region, we used the distance estimations of IRAS $05168+3634$ star-forming region: $6.08 \mathrm{kpc}$ (kinematic estimation) and $1.88_{-0.17}^{+0.21} \mathrm{kpc}$ distance (based on the trigonometric parallax).

\subsection{Selection of Objects}

For the selection of objects in the molecular cloud, we have used the data of NIR, MIR and FIR catalogs (see Section 2) within the radius of 24 arcmin concerning to conditionally selected IRAS $05168+3634$ source. As the main catalog, we have chosen GPS UKIDSS-DR6. We have selected objects with a probability of being noise $<50 \%$ and taking into account the completeness limit of UKIDSS survey in $\mathrm{K}$ band, the objects that have a measured magnitude of $K \geqslant 18.02$ have been removed from the list. This yielded approximately 48000 objects. The MIR and FIR photometric catalogs were cross-matched with GPS UKIDSS-DR6 catalog within $3 \sigma$ of combined error matching radius and then was obtained a combined photometric catalog. Thereafter, the following set of steps were followed for the identification of the young stellar objects (YSOs).

\subsection{C-C diagrams}

One of the most powerful tools for identifying YSO candidates via reddening and excess is their location on color-color diagrams. The choice of colors depends on the available data. We have constructed 4 color-color (hereafter C-C) diagrams. As YSOs we have chosen those ones which are 


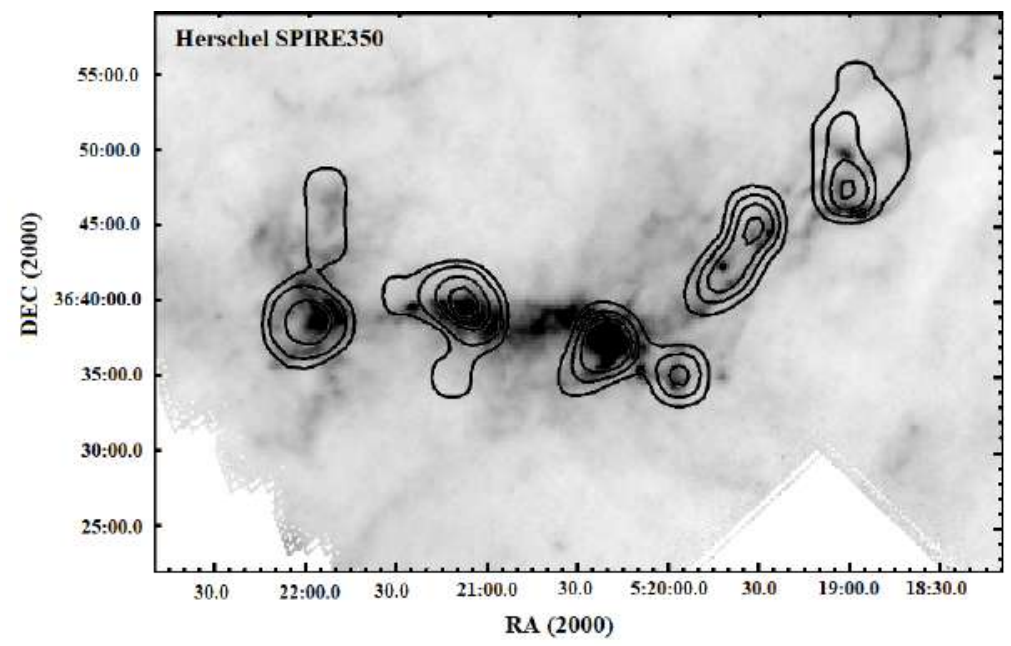

Figure 2: Stellar surface density distribution based on Herschel PACS $160 \mu \mathrm{m}$ photometry. Stellar surface density distribution map is overplotted on Herschel SPIRE $350 \mu \mathrm{m}$ image. The surface density of the last isodences exceeds the average value of the field surface density on $1 \sigma$

classified as objects with infrared excess in at least $2 \mathrm{C}-\mathrm{C}$ diagrams to minimize the likelihood of the wrong choices.

The first infrared excess objects identification was carried out with (J$\mathrm{H})$ vs. $(\mathrm{H}-\mathrm{K}) \mathrm{C}-\mathrm{C}$ diagram. Figure 3 top left panel shows the $(\mathrm{J}-\mathrm{H})$ vs. (H-K) C-C diagram where the solid and dashed curves represent the locus of the intrinsic colors of dwarf and giant stars, taken from Bessell \& Brett (1988) after being converted to the CIT system using the relations given by Carpenter (2001). The parallel solid lines drawn from the base and tip of the dwarf and giant loci, are the interstellar reddening vectors (Rieke \& Lebofsky 1985). The locus of unreddened classical T Tauri stars (CTTSs) is taken from Meyer et al. (1997). The region bounded by the dashed lines where the PMS stars with intermediate mass, i.e., Herbig Ae/Be stars are usually found (Hernández et al. 2005). The objects with different evolutionary stages have certain places in this diagram (Lada \& Adams 1992). We have selected those stars as YSOs, which have the considerable, accurately expressed IR excess i.e objects located to the right of reddening vectors.

We have used the data of GLIMPSE 360 catalog to construct C-C diagram combining NIR and MIR photometry. Figure 2 top right panel shows the $\mathrm{K}-[3.6]$ vs. [3.6]-[4.5] C-C diagram where the diagonal lines outline the region of location of YSOs with both Class I and Class II evolutionary stages. The de-reddened colors are separated into Class I and II domains by the dashed line. Arrow shows extinction vector (Flaherty et al. 2007). All the lines in the K- [3.6] vs. [3.6]-[4.5] diagram are taken from the (Allen et al. 2007). 

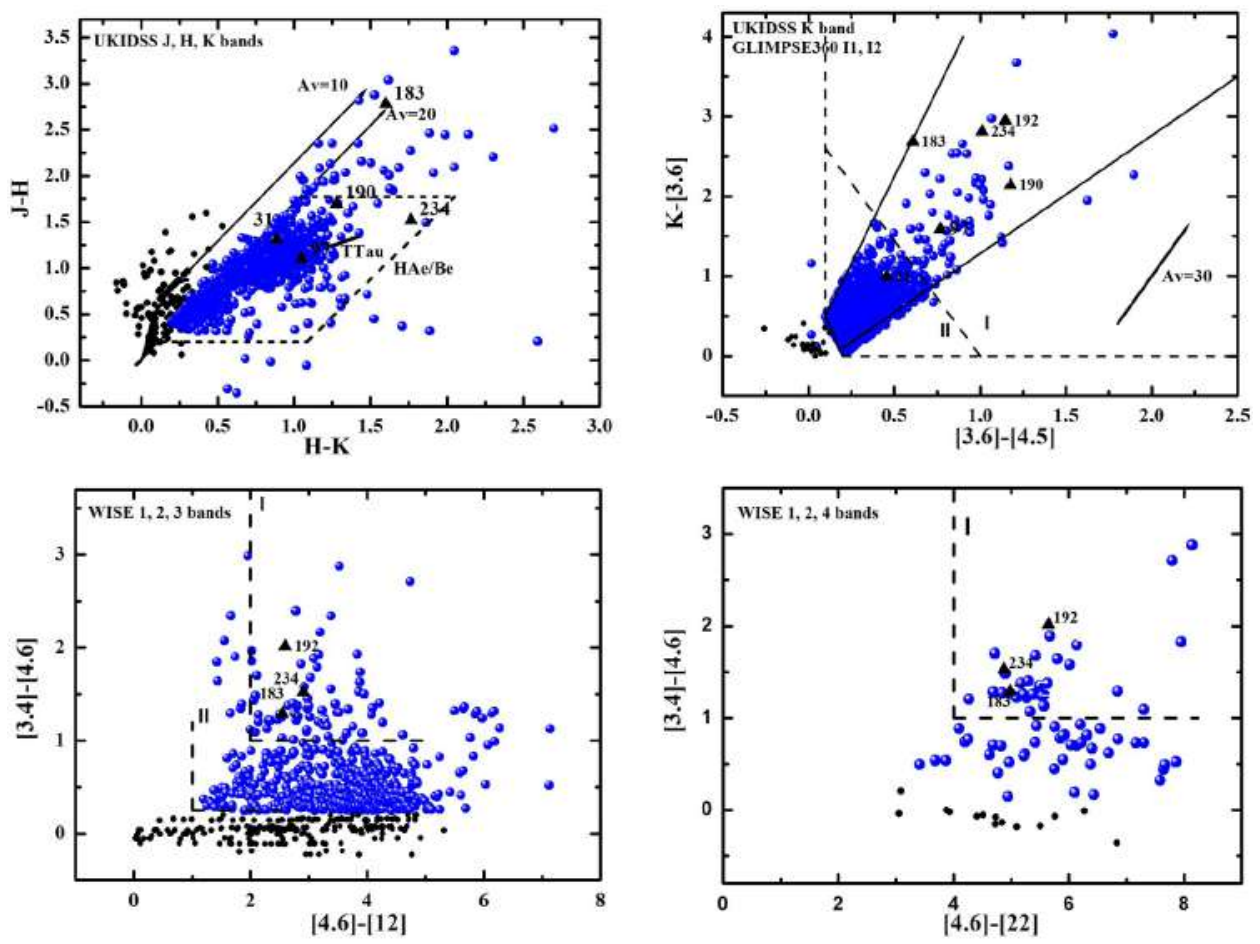

Figure 3: The C-C diagrams of the region. The blue circles are selected YSOs and black circles are unclassified ones. Not all unclassified objects are presented in these diagrams. IRAS sources are marked by triangles and they are labelled.

We have also constructed 2 other C-C diagrams using the list of objects with good WISE detection, i.e., those possessing photometric uncertainty $<0.2$ mag in WISE bands. Figure 3 below left panel shows the [3.4]-[4.6] vs. [4.6]-[12] C-C diagram and below right panel shows [3.4]-[4.6] vs. [4.6][22] $\mathrm{C}-\mathrm{C}$ diagram. As it was mentioned for previous $\mathrm{C}-\mathrm{C}$ diagrams, the objects with different evolutionary stages are located in certain places in this diagram too (Koenig et al. 2012).

Totally, we have selected 1224 YSOs within 24 arcmin radius and they are marked with blue circles. IRAS sources are marked by triangles and they are labelled in diagrams. Unfortunately, this kind of selection of YSOs cannot give a possibility to find the exact number of YSOs.

The distribution of classified YSOs in the field, shown on Figure 4, clearly shows that Class II objects are distributed more homogeneous on the field than Class I objects which are located in certain areas and show clear concentrations. Since the region is quite large, then the probability of being fore/background objects within the selected Class II objects is very high, and in case of Class I objects, that possibility is small, as they are not detected outside of the molecular cloud filaments. In order to minimize the number of incorrect Class II objects, further investigations will only be per- 


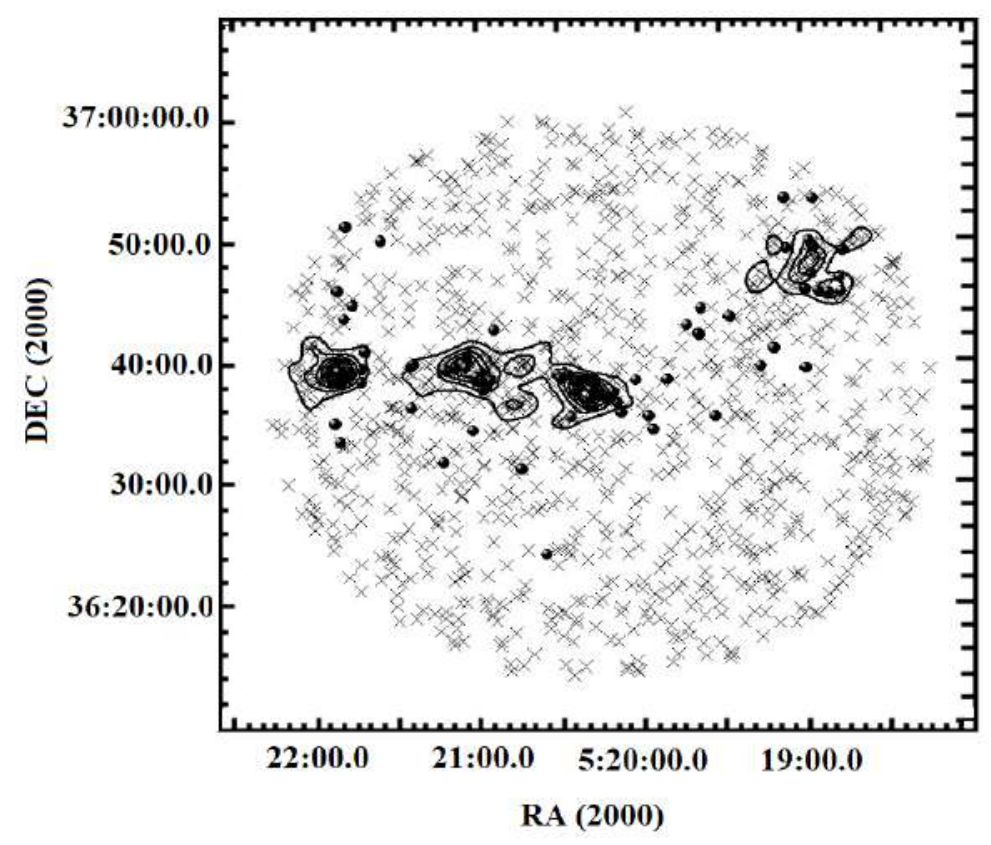

Figure 4: Stellar surface density distribution based on color-color diagrams. Class I and Class II evolutionary stage objects are marked by filled circles and crosses, respectively.

formed on concentration areas. For that purpose, we have constructed map of the distribution of stellar surface density within a $48^{\prime} \times 48^{\prime}$ region to investigate the structure and the size of each concentration in the molecular cloud, using the coordinates of selected YSOs. We have refined the radius of each sub-region relative to the geometric centers of them based on the density distribution of selected YSOs. Table 1 presents the coordinates of IRAS sources in (2)-(3) columns, in (4)-(5) columns are the coordinates of geometric centers and in last column are shown the radii based on stellar density distribution. Two objects from GPS UKIDSS-DR6 catalog were identified with IRAS $05162+3639$ source as a result of cross-match, i.e. probably IRAS $05162+3639$ source is binary object and three more Class I objects are located very close to it, but they do not show any real concentration. Therefore, the 0.25 arcmin value of radius given in the Table 1 is conditional and includes those three Class I objects and the binary associated with IRAS $05162+3639$ source. Further studies will be conducted for the 240 YSOs of 1224 selected from C-C diagrams and it is a total number of objects falling within the already-defined radii of five sub-regions. 


\begin{tabular}{|c|c|c|c|c|c|}
\hline IRAS & $\begin{array}{c}\alpha(\mathbf{2 0 0 0}) \\
(\text { hh mm ss })\end{array}$ & $\begin{array}{c}\delta(\mathbf{2 0 0 0}) \\
(\text { dd mm ss })\end{array}$ & $\begin{array}{c}\alpha(\mathbf{2 0 0 0}) \\
(\text { hh mm ss })\end{array}$ & $\begin{array}{c}\delta(\mathbf{2 0 0 0}) \\
(\text { dd mm ss })\end{array}$ & $\begin{array}{c}\text { Radius } \\
(\operatorname{arcmin})\end{array}$ \\
\hline$(1)$ & $(2)$ & $(3)$ & $(4)$ & $(5)$ & $(6)$ \\
\hline $05184+3635$ & 052153.2 & +363820.4 & 052152.6 & +363907.1 & 2.5 \\
\hline $05177+3636$ & 052109.4 & +363937.1 & 052102.8 & +363828.5 & 3.5 \\
\hline $05168+3634$ & 052016.4 & +363718.7 & 052022.3 & +363733.9 & 3 \\
\hline $05162+3639$ & 051938.4 & +364225.0 & 051938.4 & +364225.0 & 0.25 \\
\hline $05156+3643$ & 051903.6 & +364615.7 & 051904.0 & +364802.0 & 2.8 \\
\hline
\end{tabular}

Table 1: Geometric centers of sub-regions. (1)-Name of sub-regions, (2),(3)-The coordinates of IRAS sources, (4),(5)-The coordinates of geometric centers, (6)-The radius of each sub-region according to YSOs surface density distribution in the molecular cloud
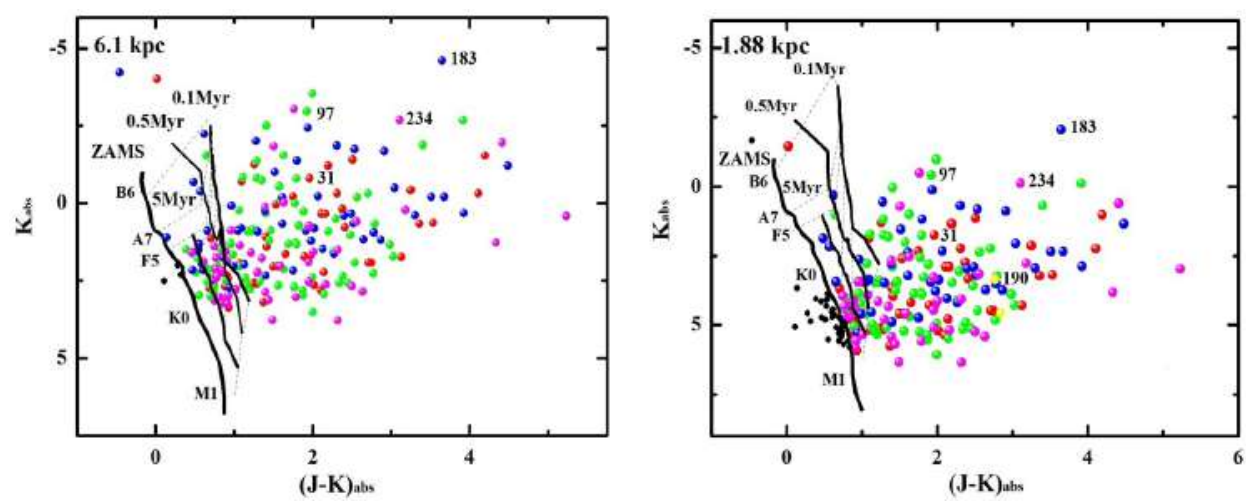

Figure 5: K vs. (J-K) C-M diagrams for the identified YSOs in sub-regions. The objects belonging to different sub-regions are marked with different colors: IRAS $05184+3635 \Rightarrow$ red, IRAS $05177+3636 \Rightarrow$ green, IRAS $05168+3634 \Rightarrow$ blue, IRAS $05162+3639 \Rightarrow$ yellow and IRAS $05156+3643 \Rightarrow$ pink. The IRAS sources are labelled.

\subsection{C-M diagrams}

The distribution of the 240 identified YSOs in the K vs. J-K colormagnitude (hereafter C-M) diagrams are shown in Figure 5 with filled circles in different colors for each sub-region. In Figure 5 the zero-age main sequence (ZAMS, the thick solid curve) and PMS isochrones (thin solid curves) are taken from Siess et al. (2000). The J and K photometry of the selected YSOs are corrected for two different distances: 6.1 and $1.88 \mathrm{kpc}$, and for interstellar extinction estimated according to the COBE/DIRBE and IRAS/ISSA maps.

In general, the selected YSOs (according to the C-C diagrams) are distributed to the right of $0.1 \mathrm{Myr}$ isochrones and this distribution confirms their being YSOs. Only a few identified as YSOs objects in the C-C diagrams are located to the left (black circles) of ZAMS, which means their non-membership, i.e. probably they are fore/background objects. 
Study of stellar population in new extended star formation region

\begin{tabular}{cccccccccc}
\hline \multirow{2}{*}{ IRAS } & \multicolumn{2}{c}{ C-C } & \multicolumn{2}{c}{ C-M-1.88 kpc } & \multicolumn{2}{c}{ C-M-6.1 kpc } & Class I & \multirow{2}{*}{$\mathrm{A}_{v}$} & $\alpha$ slope \\
& Mem. & $\begin{array}{c}\text { Class I } \\
(\%)\end{array}$ & Mem. & $\begin{array}{c}\text { Class I } \\
\text { Mem. }\end{array}$ & $\begin{array}{c}\text { Class I } \\
(\%)\end{array}$ & & $(\%)$ & & \\
\hline$(1)$ & $(2)$ & $(3)$ & $(4)$ & $(5)$ & $(6)$ & $(7)$ & $(8)$ & $(9)$ & $(10)$ \\
\hline $05184+3635$ & 52 & 21 & 48 & 23 & 52 & 21 & 11 & 1.4 & $0.12 \pm 0.04$ \\
$05177+3636$ & 79 & 28 & 65 & 34 & 79 & 28 & 22 & 1.34 & $0.2 \pm 0.02$ \\
$05168+3634$ & 57 & 43 & 45 & 54 & 56 & 45 & 24 & $4.3(4.5)$ & $0.21 \pm 0.05$ \\
$05162+3639$ & 5 & - & 5 & - & 5 & - & 5 & 1.23 & - \\
$05156+3643$ & 47 & 20 & 40 & 23 & 47 & 20 & 9 & 1.03 & $0.15 \pm 0.04$ \\
\hline
\end{tabular}

Table 2: Characterizes of sub-regions. (1)-Name of sub-regions, (2)-(3)number of YSOs and the fraction of Class I objects (in percent) according to the C-C diagrams, (4)-(7)-number of YSOs and the fraction of Class I objects (in percent) according to the C-M diagrams in different distances, (8)-number of Class I evolutionary stage objects, (9)-Interstellar extinction, (10)-The $\alpha$ slope of KLF of each sub-region

According to the results of $\mathrm{C}-\mathrm{C}$ and $\mathrm{C}-\mathrm{M}$ diagrams, one can note that within the selected radius of each sub-region the youngest is IRAS $05168+3634$ star-forming region, since Class I objects represent a fairly large percentage.

\subsection{K Luminosity function}

The luminosity function in the K-band (KLF) is frequently used in studies of young clusters and star-forming regions as a diagnostic tool of the initial mass function (IMF) and the star formation history of their stellar populations (Zinnecker et al. 1993; Lada \& Lada 1995). It is possible to construct the KLF to constrain the age of the embedded stellar population in each star-forming region independently. As pointed out by Lada et al. (1996), the age of a sub-region can be estimated by comparing its KLF to the observed KLFs of other young clusters. In the case of IRAS $05162+3639$ sub-region, the number of YSOs is not enough to construct KLF and so it is impossible to estimate the value of $\alpha$ slope. The KLFs corresponding fitted slopes are provided in Column (10) of Table 2. According to the calculation of Massi et al. (2000), $\alpha$ values between 0.2-0.28 are consistent with the ages of $0.1-3 \mathrm{Myr}$. The $\alpha$ values of the observable sub-regions are close to this range of values, therefore, the age of all four sub-regions can be estimated between 0.1-3 Myr, which also well reflects the location of stellar objects relatively to the isochrones.

\subsection{SED analysis}

We have constructed the spectral energy distributions (SEDs) for 45 Class I and 75 Class II evolutionary stage YSOs and fitted them with the radiative transfer models of Robitaille et al. (2007). This procedure has 
been done using wavelengths ranging from $1.1 \mu \mathrm{m}$ to $160 \mu \mathrm{m}$ in particular J, H, K (UKIDSS), 3.6 and $4.5 \mu \mathrm{m}$ (Spitzer IRAC), 3.4, 4.6, 12 and $22 \mu \mathrm{m}$ (WISE), 8.28, 12.13, 14.65 and $21.3 \mu \mathrm{m}$ (MSX), 12 and $25 \mu \mathrm{m}$ (IRAS) and $70,160 \mu \mathrm{m}$ (Herschel PACS). The SED fitting was carried out using both distance estimations: 1.88 and $6.1 \mathrm{kpc}$. We used the ranges of the interstellar extinction $\left(\mathrm{A}_{v}\right)$ and the distances of 1 - $40 \mathrm{mag}$ and $5.5-6.5 \mathrm{kpc}$ and $1.6-2 \mathrm{kpc}$, respectively.

The results of the SED fitting tool in general confirm the age estimations obtained by the KLF slope for each sub-region and the results obtained in the $\mathrm{C}-\mathrm{C}$ and $\mathrm{C}-\mathrm{M}$ diagrams for individual objects.

\section{Conclusion}

Our investigation shows that the IRAS $05168+3634$ star-forming region has more complicated structure in the FIR wavelengths and is located in a quite large molecular cloud within the region of $\sim 24$ arcmin radius (the center of the molecular cloud is conditionally selected IRAS $05168+3634$ source), which, in turn, consists of another four star-forming regions. From the statistical analysis follows that all IRAS star-forming regions are at the same distance: $6.1 \mathrm{kpc}$ (Molinari et al. 1996) or $1.88 \mathrm{kpc}$ (Sakai et al. 2012). In addition, these regions repeat the shape of the molecular cloud. And also the distances of IRAS $05184+3635$ and IRAS $05177+3636$ assessed based on the ${ }^{13} \mathrm{CO}$ velocities were evaluated at the same $1.4 \mathrm{kpc}$ value, which is coincides with the distance of IRAS $05168+3634$ based on trigonometric parallax.

In this paper, we analyzed the stellar content of the molecular cloud and separately for each star-forming region associated with IRAS sources using the NIR and MIR photometric data. We obtained the census of the young stellar population and their characteristics within 24 arcmin radius region surrounding the molecular cloud, which includes 1224 candidate YSOs, and 240 of them are concentrated around five IRAS sources. We have classified 71 YSOs as objects with evolutionary stage Class I and 132-169 YSOs (depending on the distance) with evolutionary stage Class II stars within radii of sub-regions. It should be noted that, unlike the Class II objects, the Class I objects are located mainly in the filaments of the molecular cloud, i.e. the distribution of Class I objects reflects the initial state of the parent molecular cloud. The estimated distances and the interstellar extinctions of each sub-region were taken into account in corrections of $\mathrm{J}$ and $\mathrm{K}$ magnitudes for $\mathrm{C}-\mathrm{M}$ diagrams which generates such a difference in the number of Class II objects.

We have also calculated the slope the KLFs for four sub-regions, namely IRAS 05184+3635, IRAS 05177+3636, IRAS 05168+3634 and IRAS 05156+3643. According to the values of the slopes of KLFs, the age of all four sub- 
regions can be estimated between 0.1-3 Myr. There are not enough YSOs in IRAS $05162+3639$ sub-region, so KLF for this sub-region were not constructed.

The SEDs are constructed for 45 Class I and 75 Class II YSOs. This procedure has been done using wavelengths ranging from $1.1 \mu \mathrm{m}$ to $160 \mu \mathrm{m}$. The results of the SED fitting tool, in general, are well correlated with the age estimations obtained by the KLF slope for each sub-region and also, at the distance of 1.88 and $6.1 \mathrm{kpc}$, IRAS sources can be classified as middle-mass YSOs which confirm the results obtained in the $\mathrm{C}-\mathrm{C}$ and $\mathrm{C}-\mathrm{M}$ diagrams.

\section{Acknowledgements}

This research has made use the data obtained at UKIRT, which is supported by NASA and operated under an agreement among the University of Hawaii, the University of Arizona and Lockheed Martin Advanced Technology Center. We gratefully acknowledge the use of data from the NASA/IPAC Infrared Science Archive, which is operated by the Jet Propulsion Laboratory, California Institute of Technology. This work was made by a research grant from the Armenian National Science and Education Fund (ANSEF) based in New York, USA.

\section{References}

Allen, L., Megeath, S. T., Gutermuth, R., et al. 2007, Protostars and Planets V, 361 Azatyan, N. M., Nikoghosyan, E. H., \& Khachatryan, K. G. 2016, Astrophysics, 59, 339 Bessell, M. S., \& Brett, J. M. 1988, PASP, 100, 1134

Carpenter, J. M. 2001, AJ, 121, 2851

Casoli, F., Dupraz, C., Gerin, M., Combes, F., \& Boulanger, F. 1986, A\&A, 169, 281

Churchwell, E., Babler, B. L., Meade, M. R., et al. 2009, PASP, 121, 213

Di Francesco, J., Johnstone, D., et al. 2008, ApJS, 175, 277

Dobashi, K. 2011, PASJ, 63, S1

Egan, M. P., Price, S. D., Kraemer, K. E., et al. 2003, VizieR Online Data Catalog, 5114

Faustini, F., Molinari, S., Testi, L., \& Brand, J. 2009, A\&A, 503, 801

Flaherty, K. M., Pipher, J. L., Megeath, S. T., et al. 2007, ApJ, 663, 1069

Fontani, F., Cesaroni, R., \& Furuya, R. S. 2010, A\&A, 517, A56

Griffin, M. J., Abergel, A., Abreu, A., et al. 2010, A\&A, 518, L3

Guan, X., Wu, Y., \& Ju, B. 2008, MNRAS, 391, 869

Harju, J., Lehtinen, K., Booth, R. S., \& Zinchenko, I. 1998, A\&AS, 132, 211

Hernández, J., Calvet, N., Hartmann, L., et al. 2005, AJ, 129, 856

Koenig, X. P., Leisawitz, D. T., Benford, D. J., et al. 2012, ApJ, 744, 130

Kumar, M. S. N., Keto, E., \& Clerkin, E. 2006, A\&A, 449, 1033

Lada, C. J., Alves, J., \& Lada, E. A. 1996, AJ, 111, 1964

Lada, C. J., \& Adams, F. C. 1992, ApJ, 393, 278

Lada, E. A., \& Lada, C. J. 1995, AJ, 109, 1682

Lépine, S., \& Shara, M. M. 2005, AJ, 129, 1483

Lucas, P. W., Hoare, M. G., Longmore, A., et al. 2008, MNRAS, 391, 136

Massi, F., Lorenzetti, D., Giannini, T., \& Vitali, F. 2000, A\&A, 353, 598

Meyer, M. R., Calvet, N., \& Hillenbrand, L. A. 1997, AJ, 114, 288

Molinari, S., Brand, J., Cesaroni, R., \& Palla, F. 1996, A\&A, 308, 573

Molinari, S., Brand, J., Cesaroni, R., Palla, F., \& Palumbo, G. G. C. 1998, A\&A, 336, 339

Pilbratt, G. L., Riedinger, J. R., Passvogel, T., et al. 2010, A\&A, 518, L1

Poglitsch, A., Waelkens, C., Geis, N., et al. 2010, A\&A, 518, L2

Rieke, G. H., \& Lebofsky, M. J. 1985, ApJ, 288, 618

Robitaille, T. P., Whitney, B. A., Indebetouw, R., \& Wood, K. 2007, ApJS, 169, 328

Ruiz-Velasco, A. E., Felli, D., Migenes, V., \& Wiggins, B. K. 2016, ApJ, 822, 101

Sakai, N., Honma, M., Nakanishi, H., et al. 2012, PASJ, 64, 108

Siess, L., Dufour, E., \& Forestini, M. 2000, A\&A, 358, 593 
Sunada, K., Nakazato, T., Ikeda, N., et al. 2007, PASJ, 59, 1185

Varricatt, W. P., Davis, C. J., Ramsay, S., \& Todd, S. P. 2010, MNRAS, 404, 661

Wang, K., Wu, Y. F., Ran, L., Yu, W. T., \& Miller, M. 2009, A\&A, 507, 369

Wright, E. L., Eisenhardt, P. R. M., Mainzer, A. K., et al. 2010, AJ, 140, 1868

Zhang, Q., Hunter, T. R., Brand, J., et al. 2005, Apj, 625, 864

Zinnecker, H., McCaughrean, M. J., \& Wilking, B. A. 1993, Protostars and Planets III, 429 